\title{
Cardiac TGR5 expression enhanced by hyperglycaemia in diabetic rats: A preclinical warning for disorders with excess bile acids
}

\author{
Feng Yu Kuo ${ }^{1,2}$, Shu Ping Lee ${ }^{1}$, Juei-Tang Cheng, ${ }^{3,4 *}$ and Ming Chang $\mathrm{Wu}^{1 *}$ \\ ${ }^{1}$ Department of Food Science, College of Agriculture, National Pingtung University of Science and Technology, 91201, Pingtung, Taiwan \\ ${ }^{2}$ Cardiovascular Center, Veterans General Hospital, Kaohsiung City, 81362, Taiwan \\ ${ }^{3}$ Department of Medical Research, Chi-Mei Medical Center, Tainan City, 71003, Taiwan \\ ${ }^{4}$ Institute of Medical Science, College of Health Science, Chang Jung Christian University, Tainan City, 71101, Taiwan
}

\begin{abstract}
Background: Diabetes is a risk factor for cardiovascular disorders. TGR5 levels are increased in cardiomyocytes exposed to hyperglycaemia. The objective of this study was to investigate the potential mechanism(s) for the increase in TGR5 levels in the hearts of diabetic rats.

Materials and methods: We used streptozotocin -induced diabetic rats (STZ rats) to assess the role of hyperglycaemia in increased cardiac TGR5 levels. The expression levels of TGR5 and signal transducer and activator of transcription 3, both its phosphorylated form (p-STAT3) and its native form (STAT3), in heart tissues were measured using Western blots.

Results: The increased levels of TGR5 and the ratio of p-STAT3 to STAT3 in cardiac tissues exposed to hyperglycaemic conditions were reversed by treating the hyperglycaemia. Additionally, the potential mechanisms of this effect were confirmed in a cultured rat cardiac cell line (H9c2) incubated in high-glucose (HG) medium to mimic the changes observed in vivo. TGR5 expression increased in parallel with the increased ratio of p-STAT3 and STAT3 in H9c2 cells exposed to HG, and these effects were reversed by treatment with stattic at a dose sufficient to inhibit STAT3. Similarly, the antioxidant tiron also produced the same effects in H9c2 cells.
\end{abstract}

Conclusion: Increased cardiac TGR5 levels in a type-1 diabetes model were related to hyperglycaemia, which produces free radicals to activate STAT3 for the higher expression of TGR5 in the heart. Therefore, an elevation in circulating bile acids from hepatic disorders and/or others shall be handled carefully in diabetic patients.

\section{Introduction}

Diabetes mellitus (DM) is a metabolic disease caused by the complex interactions of genetic, immunological and environmental factors. DM is a prime risk factor for aberrant cardiovascular function, including diabetic cardiomyopathy [1]. Diabetic cardiomyopathy is a ventricular dysfunction that occurs in diabetic patients independent of a recognized cause, such as coronary artery disease or hypertension [2].

The hallmark of diabetic cardiomyopathy is a subclinical phase associated with cellular structural abnormalities including cardiac hypertrophy, cardiac inflammation, fibrosis and increased apoptosis that initially leads to diastolic dysfunction and later to systolic dysfunction, eventually causing heart failure [3]. The link between diabetes and cardiac function is complex and multifactorial, though unclear [4]. However, the occurrence of hyperglycaemia, hyperlipidaemia, and oxidative stress in diabetes has been extensively documented and is implicated in the pathogenesis of various cardiovascular complications including cardiomyopathy [5]. Thus, among the various therapeutic strategies, antihyperglycemic, antihyperlipidemic, and antioxidant agents are useful in the prevention of cardiomyopathy in STZ-induced diabetes [6]. Hyperglycaemia is important in the pathogenesis of diabetic disorders, and lung injury by hyperglycaemia has been demonstrated in diabetic rats [7]. However, damage to the heart by hyperglycaemia is less common, except for cardiac hypertrophy [8].
Recent observations suggest the benefits of modest increases in bile acid levels on the host cardiovascular status. These effects are attributed to TGR5 activation [9]. Takeda G-protein-coupled receptor (TGR5), also known as M-BAR, GPBAR1 or BG37, is a member of the superfamily of G-protein-coupled receptors (GPCRs) that transduce signals through G proteins. TGR5 is expressed in cardiomyocytes [10]. Therefore, TGR5 activation leads to cyclic AMP (cAMP) accumulation for cardiac modulations [11]. TGR5 is also functional in rodent and human hearts $[10,12]$ and targeted by bile acids, both in health and in disease states. However, pathologic elevations in circulating bile acids $(\sim 200-300 \mu \mathrm{mol} / \mathrm{L})$, as observed in liver diseases, are toxic to the heart $[10,13]$. Therefore, higher expression of cardiac TGR5 may result in

${ }^{\star}$ Correspondence to: Juei-Tang Cheng, Department of Medical Research, Chi-Mei Medical Center, Yongkang District, Tainan City 71004, Taiwan, Tel: 8866251786, E-mail: jtcheng5503@gmail.com

Ming Chang Wu, Department of Food Science, College of Agriculture, National Pingtung University of Science and Technology, Neipu, Pingtung 91201, Taiwan, Tel.: 886-8-7703202, E-mail: globalizationwu@yahoo.com.tw

Key words: heart, hyperglycaemia, STAT3, STZ rat, TGR5

Received: April 22, 2019; Accepted: May 09, 2019; Published: May 13, 2019 
more sensitive and it shall be careful in disorders shown excess bile acids.

An activation of TGR5 in the heart by agonists induced cytoprotective changes in the heart and improved myocardial response to physiologic, inotropic, and hemodynamic stress in mice [14]. TGR5 expression was enhanced in a cardiac cell line (H9c2 cells) under hyperglycaemic conditions [15], although the potential mechanism(s) for this remain obscure. High glucose treatment increased the expression of signal transducer and activator of transcription 3 (STAT3) [16,17]. Furthermore, STAT signalling contributes to cellular transformation through the modulation of target genes [18]. However, the role of STAT3 in the hyperglycaemia-induced expression of TGR5 is still unknown. Therefore, it is of special interest to clarify the mediation of STAT3 in both H9c2 cells and the heart tissues of diabetic rats exhibiting high TGR5 expression during high glucose conditions.

\section{Materials and methods}

\section{Animals}

Male Sprague-Dawley (SD) rats weighing 250 to $280 \mathrm{~g}$ were obtained from the National Laboratory Animal Center (Taipei, Taiwan). The animals in all experiments were kept under anaesthesia with sodium pentobarbital ( $35 \mathrm{mg} / \mathrm{kg}$, i.p.) to minimize suffering. The experimental protocols were approved by the Institutional Animal Ethics Committee (103120201) at the Chi-Mei Medical Center. All experiments conformed to the Guide for the Care and Use of Laboratory Animals as well as the guidelines of the Animal Welfare Act.

\section{Induction of diabetes}

Rats fasted overnight and received a single intravenous (i.v.) injection of streptozotocin $(60 \mathrm{mg} / \mathrm{kg})$ to induce a type-1 diabetic model, as previously described [19]. Blood glucose levels were measured 3 days after STZ injection. Fasting rats with plasma glucose levels greater than $300 \mathrm{mg} / \mathrm{dl}$ were considered diabetic [20]. All experiments were performed 2 weeks after diabetes induction.

\section{Treatment with insulin or phlorizin}

The rats were randomly divided into 4 groups of 6 each as follows: a control group, STZ-induced diabetic group, insulin-treated diabetic group, and phlorizin-treated diabetic group. Briefly, STZ-induced diabetic rats were intraperitoneally (i.p.) injected with insulin (1 IU/ kg; Actrapid, Novo Nordisk, Bagsvaerd, Denmark) or phlorizin (1 $\mathrm{mg} / \mathrm{kg}$; Fluka Chemie AG, Switzerland) three times a day for 7 days, as previously described [15]. After their blood glucose levels were determined, all rats were sacrificed under anaesthesia, and their heart tissues were collected, weighed and stored at $-70^{\circ} \mathrm{C}$ for further analysis.

\section{Cell cultures}

H9c2 cells (BCRC No. 60096) were cultured in Dulbecco's modified Eagle's medium (DMEM, pH 7.2; Gibco-BRL Life Technologies, Gaithersburg, MD, USA) supplemented with $10 \%$ fetal bovine serum. The cells were passaged every 3-4 days and sub cultured when they reached $70-80 \%$ confluence 5 . To differentiate $\mathrm{H} 9 \mathrm{c} 2$ cells, the cells were switched to differentiation medium (1\% FBS-containing medium with $1 \mu \mathrm{M}$ of trans-retinoic acid, RA) for 7 days as described previously [21]. RA was added daily, and the medium was changed every other day.

After differentiation, the cells were incubated in serum-free medium with varying concentrations of $\mathrm{D}$-glucose (final concentration in the medium of 10, 20 or $30 \mathrm{mM}$ ) for 24 hours. H9c2 cells exposed to normal medium containing $5.5 \mathrm{mM}$ glucose were used for comparison. To rule out the osmotic effects of high glucose, the culture medium was supplemented with $30 \mathrm{mM}$ mannitol.

\section{Western blotting analysis}

The concentration of the total proteins was quantified by a bicinchoninic acid (BCA) protein assay kit (Thermo Scientific). Equal amounts of protein were resolved by $10 \%$ SDS-PAGE. After electrophoresis, the proteins were transferred to expanded polyvinylidene difluoride membranes (Merck Millipore, Burlington, MA, USA) and blocked with 5\% BSA in TBST for two hours. The membranes were then incubated in primary antibody specific against $\beta$-actin (1:5000; Sigma Aldrich, St. Louis, MO, United States); p-STAT3 (1:1000), STAT3 (1:1000) and TGR5 (1:1000) obtained from Abcam (Cambridge, UK). Following incubation, the membranes were washed with TBST and incubated for $1 \mathrm{~h}$ at room temperature with secondary antibodies. The blots were developed using a chemiluminescence kit (Thermo Scientific). Immunoblot densities were quantified using a laser densitometer. The optical densities of the bands corresponding to p-STAT3 (92 kDa), STAT3 (92 kDa), TGR5 (35 kDa) and $\beta$-actin $(43 \mathrm{kDa})$ were quantified using Gel-Pro Analyzer software version 4.0 (Media Cybernetics, Silver Spring, MD, USA).

\section{Real-time reverse transcription-polymerase chain reaction}

To measure the mRNA expression of each gene, the total RNA was extracted from the samples with TRIzol reagent (Carlsbad, CA, USA). Total RNA (200 ng) was reverse transcribed into cDNA with oligonucleotide primers (Roche Diagnostics, Mannheim, Germany). Real-time RT-PCR was performed using the TaqMan Universal PCR Master Mix (Thermo Scientific) in a $20 \mu$ reaction volume containing $50 \mathrm{ng}$ of cDNA. PCR was performed using a Light Cycler system (Roche Diagnostics). The relative gene expression levels were expressed as the ratio of the concentration of the target gene to that of $\beta$-actin. The primers to amplify TGR5 and $\beta$-actin are listed as follows:

\section{TGR5 F: 5'-TGGCTGCTGTGACTCTTTGA-3';}

TGR5 R: 5'-TGTGACATCATGGGTCTTGG-3';

$\beta$-actin F: 5'-CTAAGGCCAACCGTGAAAAG-3';

$\beta$-actin R: 5'-GCCTGGATGGCTACGTACA-3'.

\section{Statistical analysis}

The results are expressed as the mean \pm SEM for the number of experiments indicated in the legends of each figure. One-way ANOVA followed by a post hoc Tukey's test was applied for the comparison of multiple treatment groups. Data analysis was performed by using SPSS for Windows, version 17 (IBM, Chicago, IL, USA). $\mathrm{P}<0.05$ indicated significance.

\section{Results}

\section{TGR5 expression level in hearts of diabetic rats}

The fasting blood glucose level of untreated diabetic rats (325.4 \pm $13.8 \mathrm{mg} / \mathrm{dl}, \mathrm{N}=6)$ was significantly higher than that of normal control rats $(101.6 \pm 11.1 \mathrm{mg} / \mathrm{dl} ; \mathrm{N}=6)$. After 7 days of treatment with insulin or phlorizin, the serum glucose levels were significantly reduced to $128.7 \pm 9.9 \mathrm{mg} / \mathrm{dl}$ by insulin treatment or $148.3 \pm 13.6 \mathrm{mg} / \mathrm{dl}$ by phlorizin treatment in the diabetic rats $(\mathrm{N}=6)$. Western blot analysis revealed that the TGR5 levels and the p-STAT3 to STAT3 ratio in the heart tissues of diabetic rats were markedly increased compared with 
those in the control group (Figure 1A). These results were consistent with our previous reports [22-24]. Insulin or phlorizin treatment, at a dose sufficient to treat hyperglycaemia, reversed the changes in TGR5 (Figure 1B), p-STAT3 and STAT3 levels in the hearts of diabetic rats (Figure 1C).

\section{TGR5 and STAT3 levels in high glucose treated H9c2 cells}

The levels of TGR5, p-STAT3, and total STAT3 were further identified in $\mathrm{H} 9 \mathrm{c} 2$ cells treated with various concentrations of glucose. The mRNA level of TGR5 was markedly increased by glucose in a concentration-dependent manner compared to that in control cells (Figure 2A). The protein level of TGR5 and the ratio of p-STAT3 to total STAT3 in the H9c2 cell line were also increased by glucose in the same manner (Figure $2 \mathrm{~B}$ ). However, no significant changes in the TGR5 levels (Figure 2C) and the ratio of p-STAT3 to STAT3 (Figure 2D) were recorded after incubating with mannitol at a concentration that maintained the osmolarity of the high glucose medium indicated above in the H9c2 cell line. The possibility of hyperosmolarity-mediated changes in TGR5 levels and the ratio of p-STAT3 to STAT3 were excluded. Therefore, TGR5 expression was increased by high glucose in cardiac cells.

\section{The effects of STAT3-specific inhibitor (Stattic) on the expression levels of TGR5 in H9c2 cells}

Stattic was administered at a dose sufficient to inhibit STAT3 [25]. The increased mRNA levels of TGR5 in H9c2 cells were markedly reversed by stattic (Figure 3A). The protein level of TGR5 and the ratio of p-STAT3 to total STAT3 in the H9c2 cell line were also reduced by stattic in the same manner (Figure 3B). Quantification of the reduction in TGR5 levels (Figure 3C) or the ratio of p-STAT3 to STAT3 (Figure $3 \mathrm{D})$ in the H9c2 cell line supported the dose-dependent effects of stattic, similar to a previous report [26]. These results demonstrated that STAT3 increased TGR5 levels in H9c2 cells under high glucose conditions.

A

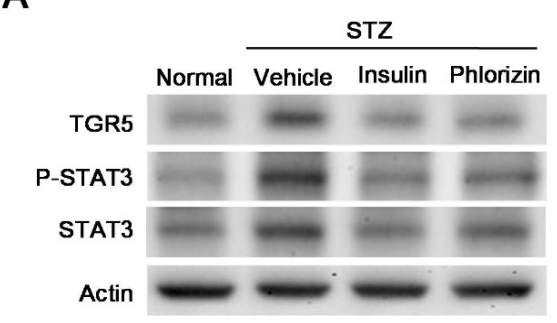

C

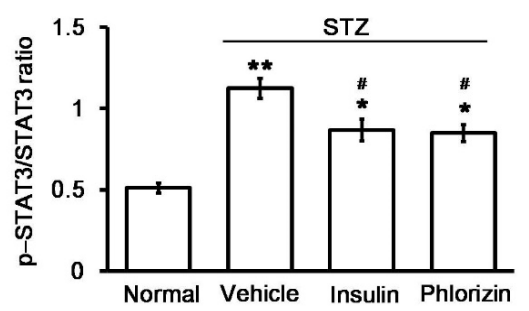

\section{The effects of antioxidant tiron on the ratio of p-STAT3 to STAT3 in addition to TGR5 levels in H9c2 cells}

To understand the role of free radicals in the activation of STAT3 phosphorylation, we used tiron at a dose sufficient to inhibit free radicals in H9c2 cells [13]. The increased mRNA levels of TGR5 in $\mathrm{H} 9 \mathrm{c} 2$ cells were reversed by tiron in a dose-dependent manner (Figure $4 \mathrm{~A})$. The protein level of TGR 5 and the ratio of p-STAT3 to total STAT3 in the H9c2 cell line were also reduced by tiron in the same manner (Figure 4B). Quantification of the reduction in TGR5 levels (Figure 4C) or the ratio of p-STAT3 to STAT3 (Figure 4D) in the H9c2 cell line supported the effects of tiron. These results demonstrate the role of free radical mediation in the increase in TGR5 levels observed in diabetic hearts.

\section{Discussion}

In the present study, we found an increased TGR5 level in the heart tissues of type 1-like diabetic rats. Insulin and phlorizin effectively lower hyperglycaemia in diabetic animals through different mechanisms; insulin decreases blood glucose through receptor-coupled signalling [27], while phlorizin inhibits renal tubular glucose reabsorption [28]. Treatment with either insulin or phlorizin for 7 days corrected hyperglycaemia and reversed changes in TGR5 levels in diabetic rats. These findings suggest that hyperglycaemia is responsible for increased TGR5 expression in heart tissues. Additionally, we treated a rat cardiac cell line (H9c2 cells) with high glucose to mimic diabetic disorders in vitro and confirmed the increase in TGR5 expression during hyperglycaemia. Moreover, the ratio of p-STAT3 to STAT3 was also changed in parallel with the TGR5 level both in vivo and in vitro. A specific inhibitor of STAT3 reversed the increased TGR 5 levels induced by high glucose in the H9c2 cells. The STAT3-specific inhibitor (stattic) also attenuated the ratio of p-STAT3 to STAT3 in the cardiac cell line, thereby indicating the regulation of TGR5 expression by STAT3.

Diabetes is a risk factor for cardiac damage, and clinical studies have indicated a possible relationship between diabetes and cardiomyopathy

B

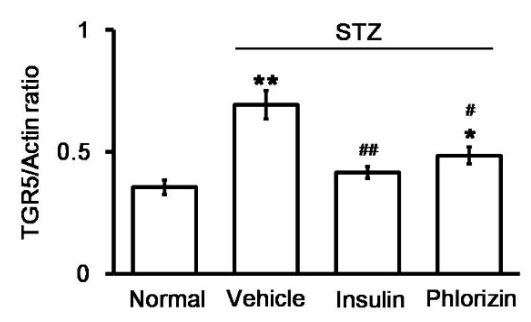

Figure 1. Changes in TGR5 expression in the hearts of diabetic rats. The representative expression of TGR5 or $\beta$-actin in addition to phosphorylated STAT3 (p-STAT3) and STAT3 (STAT3) levels (A) in cardiac tissues isolated from STZ-induced diabetic rats that received insulin or phlorizin to correct hyperglycemia, as described in the Methods section. The altered levels, expressed as the ratio of TGR5 to $\beta$-actin (B) and p-STAT3 to STAT3 levels $(\mathrm{C})$, are shown as the mean $\pm \mathrm{SEM}(\mathrm{n}=6)$ in each column. *P $<0.05$ and $* * \mathrm{P}<0.01$ vs. normal group, \#P $<$ 0.05 and \#\#P<0.01 vs. vehicle-treated control group 
A

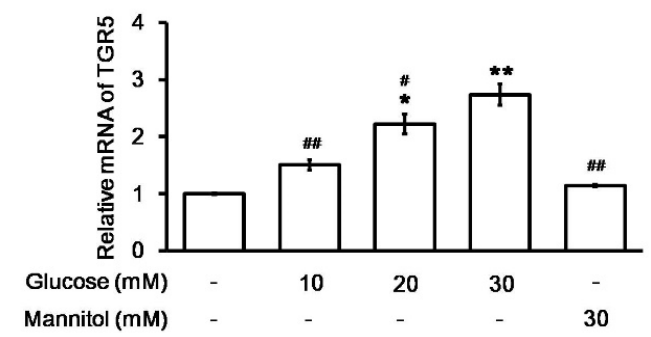

C

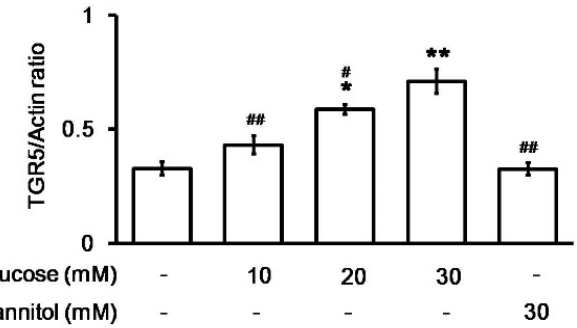

B

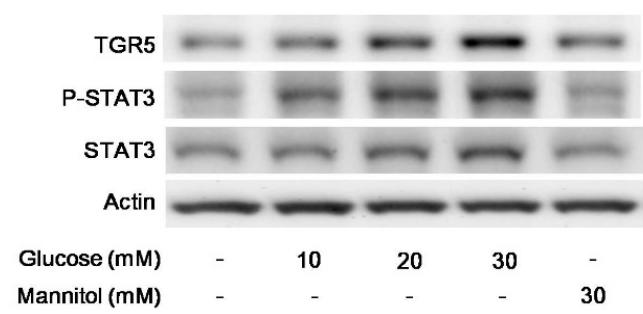

D

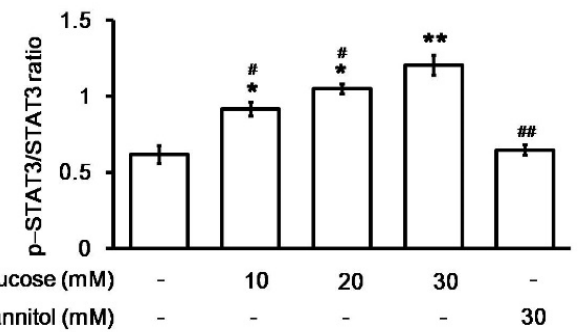

Figure 2. Changes in TGR5 or STAT3 expression in H9c2 cells after exposure to glucose at various concentrations.Changes in the mRNA levels of TGR5 in H9c2 cells incubated in medium containing the indicated concentration of glucose were compared using the mannitol-treated group as a negative control (A). The relative mRNA expression, as detected using real-time RTPCR and expressed as the ratio of TGR5 to $\beta$-actin, is indicated as the mean \pm SEM $(n=6)$. The representative expression of TGR5 or $\beta$-actin in addition to phosphorylated STAT3 ( $\mathrm{p}$-STAT3) and STAT3 (STAT3) levels in H9c2 cells exposed to medium containing various concentrations of glucose was determined using Western blotting (B). The protein levels, expressed as the ratio of TGR5 to $\beta$-actin (C) or p-STAT3 to STAT3 (D) levels, are indicated as the mean \pm SEM $(n=4)$ in each column. $* \mathrm{P}<0.05$ and $* * \mathrm{P}<0.01$ vs. values in $\mathrm{H} 9 \mathrm{c} 2$ cells exposed to normal medium (containing $5.5 \mathrm{mM}$ glucose) without the addition of glucose, \#P $<0.05$ and \# \#P $<0.01$ vs. data in $\mathrm{H} 9 \mathrm{c} 2$ cells exposed to $30 \mathrm{mM}$ glucose

A

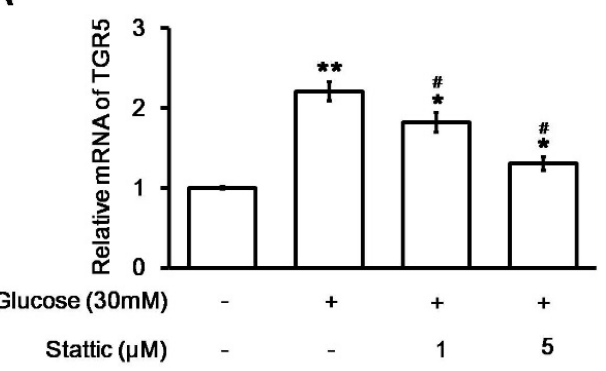

C

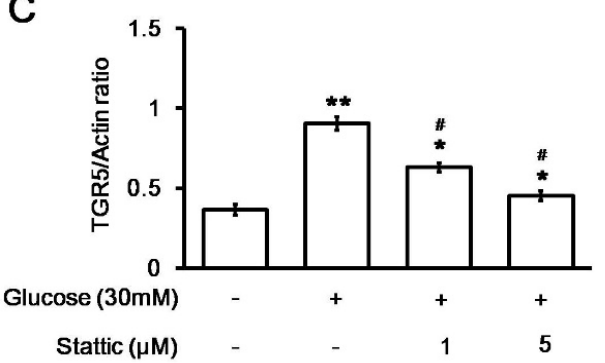

B

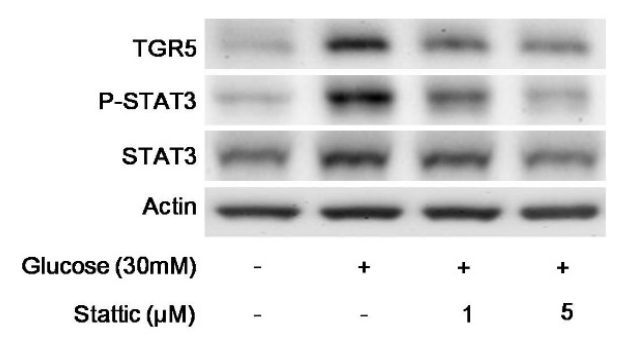

D

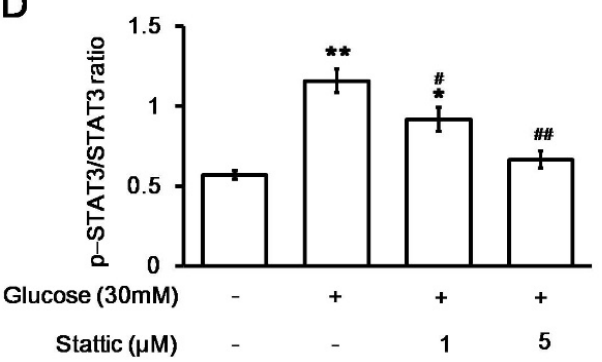

Figure 3. Effects of a STAT3 inhibitor (stattic) on changes in H9c2 cells induced by high glucose levels. Changes in the mRNA levels of TGR5 in stattic-treated (at the indicated dose) and untreated $\mathrm{H} 9 \mathrm{c} 2$ cells incubated in medium containing $30 \mathrm{mM}$ glucose were compared (A). The relative mRNA expression, as detected using real-time PCR and reported as the ratio of TGR5 to $\beta$-actin levels, is indicated as the mean \pm SEM $(n=6)$. The representative expression of TGR5 or $\beta$-actin in addition to the phosphorylated STAT3 (p-STAT3) and STAT3 (STAT3) levels in stattic-treated or untreated H9c2 cells exposed to medium containing $30 \mathrm{mM}$ glucose was also compared using Western blotting (B). The protein levels, expressed as the ratio of TGR5 to $\beta$-actin $(\mathrm{C})$ or $\mathrm{p}$-STAT3 to STAT3 levels (D), are indicated as the mean $\pm \mathrm{SEM}(\mathrm{n}=4)$ in each column. $* \mathrm{P}<0.05$ and $* * \mathrm{P}<0.01$ vs. values in $\mathrm{H} 9 \mathrm{c} 2$ cells exposed to normal medium (containing $5.5 \mathrm{mM}$ glucose), $\# \mathrm{P}<0.05$ and \# \#P $<0.01 \mathrm{vs}$. data in $\mathrm{H} 9 \mathrm{c} 2$ cells exposed to $30 \mathrm{mM}$ glucose 
A
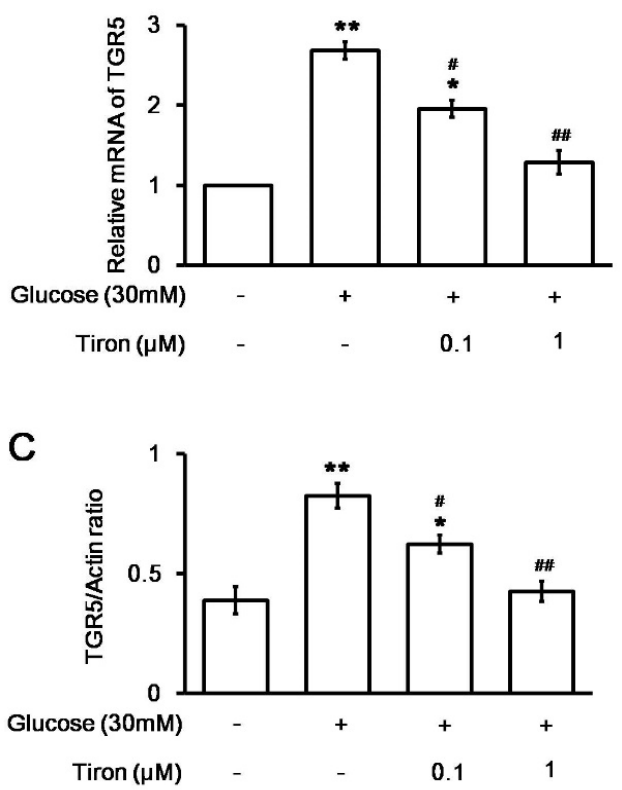

B
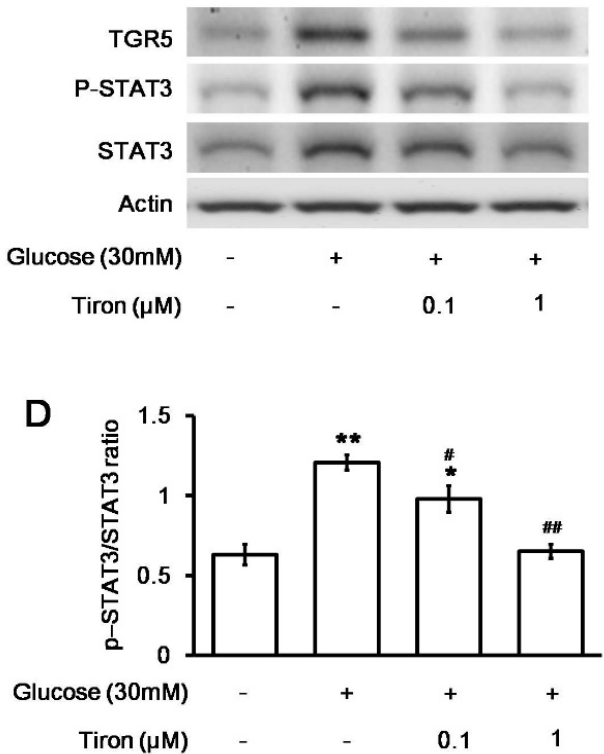

Figure 4. Effects of an antioxidant (tiron) on changes in H9c2 cells induced by high glucose. Changes in the mRNA levels of TGR5 in tiron-treated (at the indicated doses) or untreated H9c2 cells incubated in medium containing $30 \mathrm{mM}$ glucose medium were compared (A). The related mRNA expression, as detected using real-time PCR and expressed as the ratio of TGR5 to $\beta$-actin levels, is indicated as the mean \pm SEM $(\mathrm{n}=6)$. The expression of TGR5 or $\beta$-actin in addition to the phosphorylated STAT3 (p-STAT3) and STAT3 levels in tiron-treated or untreated H9c2 cells exposed to medium containing $30 \mathrm{mM}$ glucose was also compared using Western blotting (B). The protein levels, expressed as the ratio of TGR5 to $\beta$-actin (C) or p-STAT3 to STAT3 (D) levels, are indicated as the mean \pm SEM $(n=4)$ in each column. $* \mathrm{P}<0.05$ and $* * \mathrm{P}<0.01$ vs. values in H9c2 cells exposed to normal medium (containing 5.5 mM glucose), \#P $<0.05$ and \# \#P $<0.01$ vs. data in $\mathrm{H} 9 \mathrm{c} 2$ cells exposed to $30 \mathrm{mM}$ glucose

[29], although the complicated mechanism(s) of this relationship are still unclear. Diabetes is primarily characterized by hyperglycaemia. TGR5 has been implicated in the maintenance of cardiac function [30]. However, the effect of high glucose on TGR5 levels has not been described. In the present study, we discovered that the TGR5 level was increased in the hearts of diabetic rats and that this increase was markedly reversed through the correction of blood glucose levels. Therefore, hyperglycaemia induced TGR5 expression was identified in the hearts of diabetic rats. STAT3 is a cytoplasmic transcription factor that transmits extracellular signals to the nucleus [31]. Activated STAT3 in the nucleus binds to specific DNA promoter sequences to regulate gene expression [32]. Hyperglycaemia increases STAT3 activation, thereby contributing to the pathophysiology of tissue injury [22]. STAT3 activation and an increased ratio of phosphorylated STAT3 (p-STAT3) to STAT3 may promote nuclear translocation. Moreover, p-STAT3 was induced at Y705 and S727 in cells with the activation of STAT3 by high glucose levels [33]. Therefore, in the current study, we focused on changes in the ratio of p-STAT3 to STAT3. Our data also demonstrated that the increased ratio of p-STAT3 to STAT3 was reversed with the correction of hyperglycaemia in the hearts of diabetic rats. Additionally, similar changes were observed in the cultured cardiac cell line (H9c2 cells) that was induced by high glucose levels. Moreover, a specific inhibitor of STAT3 in H9c2 cells markedly reversed the increased TGR5 levels triggered by high glucose. The pharmacological inhibitor specific to STAT3 (stattic) also reversed the changes in the ratio of p-STAT3 to STAT3. Therefore, the increased TGR5 level was regulated by activated STAT3. The specificity of stattic has been criticized $[34,35]$. However, stattic has been widely used as a specific STAT3 inhibitor in numerous studies [36,37] As evidenced by our findings, hyperglycaemia may increase STAT3 activation to enhance TGR5 expression, which could be a possible mechanism underlying cardiac disorders in diabetes.
STATs belong to a group of transcription factors that are activated by free radicals [38], and the activation of STAT3 is closely correlated with the occurrence of fibrotic diseases [39]. Free radicals and/or oxidative stress are associated with hyperglycaemia [40]. Therefore, the activation of STAT3 is mainly induced by free radicals from hyperglycaemia. In this report, we found that the well-known free radical inhibitor tiron effectively reversed increased TGR5 levels in a cardiac cell line. Therefore, free radicals produced from hyperglycaemia may activate STAT3 to promote TGR5 expression in the heart during diabetes. Therefore, an elevation in the levels of circulating bile acids in diabetic patients with hepatic disorders or others shall be examined carefully.

Animal models of diabetic complications in the heart that show high TGR5 expression and simulate functional changes in humans were not applied in the current study. Therefore, the increase in TGR5 levels in diabetic cardiomyopathy needs more detailed study in the future. Additionally, the inhibition of STAT3 using siRNA 1 would be more specific than the use of a pharmacological inhibitor. This is another limitation of this report and should be investigated in the future.

\section{Conclusion}

Taken together, our data demonstrated for the first time that free radicals produced from hyperglycaemia may activate STAT3 to promote TGR5 expression in the hearts of diabetic rats. Our findings highlight the potential mechanism(s) of diabetes-induced cardiac complications. Additionally, the current findings also suggest that the cardiac TGR5 response is more sensitive in hyperglycaemic conditions than in normoglycemic conditions. Therefore, an elevation in circulating bile acids from hepatic disorders and/or others shall be handled carefully in diabetic patients. Moreover, the application of TGR5 ligands or agonists 
should be carried out with care during hyperglycaemia both in vivo and in vitro.

\section{Acknowledgements}

We thank Y.L. Yen and Y.C. Chen for kind help in the experiments and we acknowledge American Journal Expert (AJE) for editing assistance.

\section{Author contribution}

The author(s) have made the following declarations about their contributions: MCW conceived and designed the experiments, FYK and SPL performed the experiments, FYK analyzed the data, JTC and MCW contributed reagents/materials/analysis tools. FYK and JTC wrote the manuscript.

\section{Funding}

The present study was supported by a grant from the Ministry of Science and Technology (MOST 104-2320-B-384-004-MY3) in Taiwan, the Republic of China.

\section{Availability of data and materials}

The datasets supporting the conclusions of this article are included within the article. Any request of data and material may be sent to the corresponding author.

\section{Competing interests}

The authors declare that they have no competing interests.

\section{Consent for publication}

Not applicable.

\section{Ethics approval and consent to participate}

All animal experimental protocols were approved by the ethics committee (Institutional Animal Care and Use Committee) of Chi-Mei Medical Center (Tainan, Taiwan).

\section{References}

1. Parmar K, Prajapati A, Patel S, Patel M, Patel B (2012) Cardioprotective effects of lisinopril in streptozotocin induced type-II diabetic rats. Acta Endocrinologica (Buc) 8: $177-188$.

2. Palmieri V, Capaldo B, Russo C, Iaccarino M, Pezzullo S, et al. (2008) Uncomplicated type 1 diabetes and preclinical left ventricular myocardial dysfunction: Insights from echocardiography and exercise cardiac performance evaluation. Diabetes Res Clin Pract 79: 262-268

3. Kayama Y, Raaz U, Jagger A, Adam M, Schellinger IN, et al. (2015) Diabetic cardiovascular disease induced by oxidative stress. Int J Mol Sci 16: 25234-25263.

4. Dokken BB (2008) The pathophysiology of cardiovascular disease and diabetes: Beyond blood pressure and lipids. Diabetes Spectrum 21: 160-165.

5. Rajesh M, Mukhopadhyay P, Batkai S, Patel V, Saito K, et al. (2010) Cannabidiol attenuates cardiac dysfunction, oxidative stress, fibrosis, and inflammatory and cell death signalling pathways in diabetic cardiomyopathy. $J$ Am Coll Cardiol 56: 21152125.

6. Patel SS, Goyal RK (2011) Cardioprotective effects of gallic acid in diabetes-induced myocardial dysfunction in rats. Pharmacognosy Res 3: 239-245.

7. Wang CM, Hsu CT, Niu HS, Chang CH, Cheng JT, et al. (2016) Lung damage induced by hyperglycemia in diabetic rats: The role of signal transducer and activator of transcription 3 (STAT3). J Diabetes Complications 30: 1426-1433. [Crossref]

8. Cheng KC, Chang WT, Li Y, Cheng YZ, Cheng JT, et al. (2018) GW0742 activates peroxisome proliferator-activated receptor delta to reduce free radicals and alleviate cardiac hypertrophy induced by hyperglycemia in cultured H9c2 cells. J Cell Biochem 119: $9532-9542$
9. Ibrahim E, Diakonov I, Arunthavarajah D, Swift T, et al. (2018) Bile acids and their respective conjugates elicit different responses in neonatal cardiomyocytes: role of $\mathrm{Gi}$ protein, muscarinic receptors and TGR5. Sci Rep 8: 7110. [Crossref]

10. Desai MS, Shabier Z, Taylor M, Lam F, Thevananther S, et al. (2010) Hypertrophic cardiomyopathy and dysregulation of cardiac energetics in a mouse model of biliary fibrosis. Hepatology 51: 2097-2107.

11. Tiwari A, Maiti P (2009) TGR5: an emerging bile acid G-protein-coupled receptor target for the potential treatment of metabolic disorders. Drug Discov Today 14: 523530

12. Desai MS, Penny DJ (2013) Bile acids induce arrhythmias: old metabolite, new tricks. Heart 99: 1629-1630. [Crossref]

13. Desai MS, Mathur B, Eblimit Z, Vasquez H, Taegtmeyer H, et al. (2017) Bile acid excess induces cardiomyopathy and metabolic dysfunctions in the heart. Hepatology 65: 189-201. [Crossref]

14. Eblimit Z, Thevananther S, Karpen SJ, Taegtmeyer H, Moore DD, et al. (2018) TGR5 activation induces cytoprotective changes in the heart and improves myocardia adaptability to physiologic, inotropic, and pressure-induced stress in mice. Cardiovasc Ther 36: e12462. [Crossref]

15. Cheng KC, Chang WT, Kuo FY, Chen ZC, et al. (2019) TGR5 activation ameliorates hyperglycemia-induced cardiac hypertrophy in H9c2 cells. Sci Rep 9: 3633. [Crossref]

16. Wang L, Li J, Li D (2015) Losartan reduces myocardial interstitial fibrosis in diabetic cardiomyopathy rats by inhibiting JAK/STAT signalling pathway. Int J Clin Exp Pathol 8: 466-473.

17. Yeh MC, Chen LJ, Niu HS, Yang TT, Lin KC, et al. (2014) Signals for increase of $\mu$-opioid receptor expression in muscle by hyperglycemia. Neurosci Lett 582: 109-114. [Crossref]

18. Cimica V1, Chen HC, Iyer JK, Reich NC (2011) Dynamics of the STAT3 transcription factor: nuclear import dependent on Ran and importin- $\hat{I}^{2} 1$. PLoS One 6: e20188. [Crossref]

19. Cheng JT, Huang CC, Liu IM, Tzeng TF, Chang CJ (2006) Novel mechanism for plasma glucose-lowering action of metformin in streptozotocin-induced diabetic rats. Diabetes 55: 819-825.

20. Kuo SC, Chung HH, Huang CH, Cheng JT (2014) Decrease of hyperglycemia by syringaldehyde in diabetic rats. Horm Metab Res 46: 8-13. [Crossref]

21. Kankeu C, Clarke K, Van Haver D, Gevaert K, Impens F, et al. (2018) Quantitative proteomics and systems analysis of cultured $\mathrm{H} 9 \mathrm{C} 2$ cardiomyoblasts during differentiation over time supports a 'function follows form' model of differentiation. Mol Omics 14: 181-196. [Crossref]

22. Chen Y, Wang JJ, Li J, Hosoya KI, Ratan R, et al. (2012) Activating transcription factor 4 mediates hyperglycaemia-induced endothelial inflammation and retinal vascular leakage through activation of STAT3 in a mouse model of type 1 diabetes. Diabetologia 55: 2533-2545.

23. Cheng MF, Chen LJ, Wang MC, Hsu CT, Cheng JT (2014) Decrease of FGF receptor (FGFR) and interstitial fibrosis in the kidney of streptozotocin-induced diabetic rats. Horm Metab Res 46: 1-7. [Crossref]

24. Fong Y, Shen KH, Chen LJ, Cheng JT (2011) Changes of CCAAT enhancer-binding proteins (CEBPs) in the lung of streptozotocin-induced diabetic rats. Horm Metab Res 43: $261-267$.

25. Watson AJ, Gao L, Sun L, Tsun J, Jabbour A, et al. (2013) Enhanced preservation of the rat heart after prolonged hypothermic ischemia with erythropoietin supplemented Celsior solution. J Heart Lung Transplant 32: 633-640.

26. Kuo SC, Li Y, Cheng YZ, Lee WJ, Cheng JT, et al. (2018) Molecular mechanisms regarding potassium bromateâ€'induced cardiac hypertrophy without apoptosis in H9c2 cells. Mol Med Rep 18: 4700-4708. [Crossref]

27. Hawkins M, Hu M, Yu J, Eder H, Vuguin P, et al. (1999) Discordant effects of glucosamine on insulin-stimulated glucose metabolism and phosphatidylinositol 3-kinase activity. J Biol Chem 274: 31312-31319.

28. Huang CJ1, Liu IM, Cheng JT (2007) Increase of peroxisome proliferator-activated receptor delta gene expression in the lungs of streptozotocin-induced diabetic rats. Pulm Pharmacol Ther 20: 69-74. [Crossref]

29. Isfort M, Stevens SC, Schaffer S, Jong CJ, Wold LE (2014) Metabolic dysfunction in diabetic cardiomyopathy. Heart Fail Rev 19: 35-48

30. Desai MS, Eblimit Z, Karpen SJ, Moore DD, Penny DJ (2014) Abstract 18219: TGR5 - a Novel membrane receptor for bile acids regulates myocardial response to exercise, catecholamine and pressure overload induced stress. Circulation 130: A18219-A18219. 
31. Miller AM, Wang H, Bertola A, Park O, Horiguchi N, et al. (2011) Inflammationassociated interleukin-6/signal transducer and activator of transcription 3 activation ameliorates alcoholic and nonalcoholic fatty liver diseases in interleukin-10-deficient mice. Hepatology 54: 846-856.

32. Jung JE, Lee HG, Cho IH, Chung DH, Yoon SH, et al. (2005) STAT3 is a potential modulator of HIF-1-mediated VEGF expression in human renal carcinoma cells FASEB J 19: 1296-1298.

33. Saengboonmee C, Seubwai W, Pairojkul C, Wongkham S (2016) High glucose enhances progression of cholangiocarcinoma cells via STAT3 activation. Sci Rep 6: 18995. [Crossref]

34. Heidelberger S, Zinzalla G, Antonow D, Essex S, Basu BP, et al. (2013) Investigation of the protein alkylation sites of the STAT3:STAT3 inhibitor Stattic by mass spectrometry. Bioorg Med Chem Lett 23: 4719-4722.

35. Szelag M, Sikorski K, Czerwoniec A, Szatkowska K, Wesoly J, et al. (2013) In silico simulations of STAT1 and STAT3 inhibitors predict SH2 domain cross-binding specificity. Eur J Pharmacol 720: 38-48. [Crossref]
36. Boengler K, Ungefug E, Heusch G, Schulz R (2013) The STAT3 inhibitor stattic impairs cardiomyocyte mitochondrial function through increased reactive oxygen species formation. Curr Pharm Des 19: 6890-6895.

37. Sanseverino I, Purificato C, Gauzzi MC, Gessani S (2012) Revisiting the specificity of small molecule inhibitors: the example of stattic in dendritic cells. Chem Biol 19: $1213-1214$

38. McCormick J, Barry SP, Sivarajah A, Stefanutti G, Townsend PA, et al. (2006) Free radical scavenging inhibits STAT phosphorylation following in vivo ischemia/ reperfusion injury. FASEB $J$ 20: 2115-2117.

39. Ma X, Chen R, Liu X, Xie J, Si K, et al. (2013) Effects of matrine on JAK-STAT signalling transduction pathways in bleomycin-induced pulmonary fibrosis. Afr J Tradit Complement Altern Med 10: 442-448.

40. Pickering RJ1, Rosado CJ1, Sharma A2, Buksh S2, Tate M3, et al. (2018) Recent novel approaches to limit oxidative stress and inflammation in diabetic complications. Clin Transl Immunology 7: e1016. [Crossref]

Copyright: ( 2019 Kuo FY. This is an open-access article distributed under the terms of the Creative Commons Attribution License, which permits unrestricted use, distribution, and reproduction in any medium, provided the original author and source are credited. 